\title{
Take, Gunnar: Forschen für den Wirtschaftskrieg. Das Kieler Institut für Weltwirtschaft im Nationalsozialismus (Jahrbuch für Wirtschaftsgeschichte, Beihefte 25), 538 S., De Gruyter, Berlin u.a. 2019.
}

\section{Alexander Leipold}

Angenommen: 29. April 2021 / Online publiziert: 22. Mai 2021

(C) Der/die Autor(en) 2021

Wissenschaftliche Expertise im Allgemeinen und wirtschaftspolitische Beratung im Speziellen sind regelmäßig Gegenstand von Kontroversen. Gunnar Take legt mit seiner 2019 publizierten Dissertation über das Kieler Institut für Weltwirtschaft (IfW) einen Baustein zum Verständnis der Rolle von Experten in der nationalsozialistischen Diktatur vor. Erkenntnisleitend ist die Frage, inwieweit die Institutsmitarbeiter - nach Take überwiegend Männer (S. 6) - Handlungsspielräume in der Diktatur zum Widerstand nutzten. Die Antwort fällt negativ aus: nicht nur, dass es einen solchen Widerstand nicht gab, vielmehr verfolgte das Institut eine willfährige Anpassung an den Informationsbedarf der NS-Bürokratie und sanktionierte mit der zwischenzeitlichen Ausrichtung der Außenwirtschafts- und Versorgungsforschung am ,,völkischen Optimum“ (S. 102) auch ideologisch deren Großmachtvorstellungen. Opportunismus und Indienstnahme der Wissenschaft für die Zwecke des NS-Staates wurden nach dem Krieg nicht aufgearbeitet, sondern von wechselnden Institutsleitungen übergangen. Schon wenige Monate nach Kriegsende bereitete man Auftragsgutachten für die Landes- und Bundespolitik vor, ein weit gefächertes Netz zwischen Wissenschaft, Politik und Verwaltung besorgte die notwendigen personellen Kontinuitäten. In den 1950er Jahren sah man sich ,wieder hervorragend aufgestellt“ (S. 470).

Während die Bedeutung von wirtschaftspolitischer Expertise in den vergangenen fünfzehn Jahren in zahlreichen Studien mit der Herausarbeitung einer genuinen „Expertenkultur“ für die westdeutsche Nachkriegsgeschichte beantwortet werden konnte, kritisiert Take in der Auseinandersetzung mit den wirtschaftswissenschaftlichen Instituten vor 1945 eine ,geringe Qualität des bisherigen Forschungsstands“ (S. 5). Sein Erkenntnisinteresse richtet er auf einen gemeinsam geteilten Kern an Überzeugungen unter den Mitarbeitern. Damit knüpft er an wissenssoziologische Forschungen an, in denen die Bedeutung von epistemischen Gemeinschaften herausgestellt wird. Die Frage wird sodann von fünf Problemkomplexen angeleitet, in denen die Bedeutung externer Ereignisse, die Herausbildung autonomer Lehrmei-

\footnotetext{
A. Leipold $(\square)$

Leuphana Universität Lüneburg, Lüneburg, Deutschland

E-Mail: alexander.leipold@leuphana.de
} 
nungen innerhalb der wirtschaftswissenschaftlichen Gemeinde, die Fortentwicklung der Forschungsmethoden, der politische Einfluss durch Beratung und die handlungsleitenden Motive der Akteure während der Diktatur erörtert werden (S. 3f.).

Nach Take hat Institutsgründer Bernhard Harms mit seinem wissenschaftlichen Selbstverständnis eine Grundlage für die spätere Anpassungsbereitschaft während der Zeit des Nationalsozialismus gelegt, wenn er im Nachgang zum Werturteilsstreit den ökonomischen Sachverstand als apolitischen Beitrag zur Aufklärung deutete. Die in Kiel ausgebildeten Doktoranden, so Take, ,gingen von einer völligen Verantwortungsfreiheit der Wissenschaft aus“ (S. 472). Neben Willfährigkeit erkennt der Autor in der stetig wachsenden Institutsbibliothek und dem Wirtschaftsarchiv begünstigende Standortfaktoren. Eine auf Effizienz und Anwendungsorientierung geprägte Arbeitsweise der wissenschaftlichen Mitarbeiter ermöglichte die Erstellung von Auftragsgutachten im Schnellverfahren für aufkommende Bereichsprobleme vor und während des Zweiten Weltkriegs. Hauptabnehmer dieser Gutachten waren anfänglich das Reichsernährungsministerium und Unternehmen wie die GlanzstoffFabriken, später auch Volkswagen und mit Beginn des Krieges dann weit überwiegend das Wehrwirtschaftsamt und private Auftraggeber wie die Reichsgruppe Industrie oder die Kontinentale Öl (S. 122f.).

Take greift auf Quellenmaterial der Zentralbibliothek für Wirtschaftswissenschaften (ZBW) in Kiel und des IfW-Hausarchivs zurück. Ferner hat er Unterlagen aus dem Bundesarchiv, dem Landesarchiv Schleswig-Holstein, dem Politischen Archiv des Auswärtigen Amtes, zwei Stadtarchiven, dem Staatlichen Militärarchiv in Moskau und dem Rockefeller Archive konsultiert. Insgesamt wurden Materialien aus 16 Archiven und wissenschaftlichen Einrichtungen gesichtet. Neben thematischer Narration stützt sich der Autor auf eine analytische Darstellung des Quellenmaterials, wovon zahlreiche instruktive Abbildungen und Tabellen zeugen. Hervorgehoben seien die Übersicht über die wichtigsten Forschungsgruppen des Instituts zwischen 1934 und 1944 (S. 127), die organisatorische Einbindung der Ernährungsstatistischen Abteilung des IfW in die Autarkiepolitik von 1932 bis 1935 (S. 209) und die Auftragsarbeiten für militärische Stellen zwischen 1938 bis 1940 im Vorfeld des Überfalls der Wehrmacht auf Norwegen (S. 343f.). Take verbindet Chronologie und Ereignisgeschichte, was sich auf die Kapitelgliederung auswirkt und die Lektüre nicht immer erleichtert. Das Inhaltsverzeichnis ist detailliert genug, dem Leser die erforderliche Orientierung zu geben. Dennoch wäre ein Sachwortregister wünschenswert gewesen, um gezielt nachschlagen zu können. Hier wird man, wenn möglich, auf die digitale Ausgabe des Buches zurückgreifen müssen.

Die Studie ist in verschiedener Hinsicht anschlussfähig: Takes Organisationsgeschichte des IfW unterstreicht einmal mehr die lange Vorgeschichte der Verwissenschaftlichung des Sozialen quer zu politischen Herrschaftssystemen. Die Rekonstruktion des Forschungsschwerpunktes der Raumwirtschaftslehre stellt ein Bindeglied zur Geschichte des neoliberalen Denkkollektivs dar, für das der spätere IfWPräsident und Vorsitzende des Sachverständigenrats zur Begutachtung der gesamtwirtschaftlichen Entwicklung, Herbert Giersch, maßgeblich wurde. Und schließlich dokumentiert die Arbeit intellektuelle Pfadabhängigkeiten im Zuge der erzwungenen wirtschaftswissenschaftlichen Emigration (Gerhard Colm, Hans Neisser und andere), womit sie nicht nur die ökonomische Debatte nach dem Krieg verstehen hilft, 
sondern auch vergessene ökonomische Planungstheorien in Erinnerung ruft. Nach dem Krieg kehrte keiner der Vertriebenen nach Kiel zurück.

Funding Open Access funding enabled and organized by Projekt DEAL.

Open Access Dieser Artikel wird unter der Creative Commons Namensnennung 4.0 International Lizenz veröffentlicht, welche die Nutzung, Vervielfältigung, Bearbeitung, Verbreitung und Wiedergabe in jeglichem Medium und Format erlaubt, sofern Sie den/die ursprünglichen Autor(en) und die Quelle ordnungsgemäß nennen, einen Link zur Creative Commons Lizenz beifügen und angeben, ob Änderungen vorgenommen wurden.

Die in diesem Artikel enthaltenen Bilder und sonstiges Drittmaterial unterliegen ebenfalls der genannten Creative Commons Lizenz, sofern sich aus der Abbildungslegende nichts anderes ergibt. Sofern das betreffende Material nicht unter der genannten Creative Commons Lizenz steht und die betreffende Handlung nicht nach gesetzlichen Vorschriften erlaubt ist, ist für die oben aufgeführten Weiterverwendungen des Materials die Einwilligung des jeweiligen Rechteinhabers einzuholen.

Weitere Details zur Lizenz entnehmen Sie bitte der Lizenzinformation auf http://creativecommons.org/ licenses/by/4.0/deed.de.

\section{Ullrich, Volker: Acht Tage im Mai. Die letzte Woche des Dritten Reiches, 317 S., Beck, München 2020.}

\section{Wolfgang G. Schwanitz}

Angenommen: 29. April 2021 / Online publiziert: 7. Juni 2021

(c) Gesellschaft zur wissenschaftlichen Förderung politischer Literatur e.V. and the Author(s) 2021

Nach halb vier öffnete Diener Heinz Linge die Tür zu Adolf Hitlers Raum. Es ist passiert, wandte er sich Reichsleiter Martin Bormann zu. Auf dem Sofa saß Hitler, Kopf leicht nach vorn geneigt. Seine rechte Schläfe zeigte den Einschuss, wo Blut die Wange herablief. Die Pistole war ihm entglitten. Daneben saß Eva Braun. Ihr Geruch wies auf eine Zyankalikapsel hin. „Der Führer ist tot!“”, rief Adjutant Otto Günsche den Wartenden am 30. April 1945 im Tiefbunker unter der Alten Reichskanzlei zu. Indes hagelte es Artillerie-Granaten über dem Regierungssitz. Wie es Hitler befohlen hatte, verbrannten Helfer seine und Evas Leiche vor dem Ausgang. Abends vergruben sie zwei SS-Leute in einer Mulde im Kanzlei-Garten.

W. G. Schwanitz (ه)

Philadelphia/PA, USA

E-Mail: 130wgs@gmail.com 